PROCEEDINGS OF THE

AMERICAN MATHEMATICAL SOCIETY

Volume 133, Number 12, Pages 3613-3618

S 0002-9939(05)07942-6

Article electronically published on June 7, 2005

\title{
STRONG PSEUDO-CONTRACTIONS PERTURBED BY COMPACT OPERATORS IN BANACH SPACES
}

\author{
CLAUDIO H. MORALES
}

(Communicated by Joseph A. Ball)

\begin{abstract}
Let $X$ be a (real) Banach space, let $D$ be an open subset of $X$, and let $\mathcal{B}(X)$ denote the collection of all nonempty bounded and closed subsets of $X$. Suppose $T$ is continuous from $\bar{D}$ into $\mathcal{B}(X)$ with respect to the Hausdorff metric and strongly pseudo-contractive, while $g$ is compact from $\bar{D}$ into $X$. Then $T+g$ has a fixed point if it satisfies the classical Leray-Schauder condition on the boundary of $D$.
\end{abstract}

\section{INTRODUCTION}

The study of compact perturbations of accretive type operators has been extensively studied for the last three decades. In fact, these operators arise while solving boundary value problems, as well as eigenvalue problems. An early discussion on the existence of zeros under various types of boundary conditions on the domain of the operator can be found in Browder [1], and later in Kartsatos ([4, 5]) and Hirano [3. Our purpose here is to bring a different approach to establish a fixed point theorem for a multivalued strongly pseudo-contractive mapping perturbed by a compact operator under the well-known Leray-Schauder condition (see [11] for related results). Perhaps the generality of this condition has generated an extensive study of it in connection with an ample number of different types of nonlinear operators. Indeed, this writer has proven (see [10]) that a continuous strongly pseudo-contractive mapping under this boundary condition has a unique fixed point.

Let $X$ be a (real) Banach space. An operator $T: D(T) \subset X \rightarrow 2^{X}$ is said to be strongly pseudo-contractive (with constant $k \in(0,1)$ ) if for each $u, v \in D(T)$ there exists $j \in J(u-v)$ such that

$$
\langle u-v, j\rangle \leq k\|u-v\|^{2}
$$

for $u \in T(x)$ and $v \in T(y)$. The mapping $J: X \rightarrow X^{*}$ denotes the normalized duality mapping which is defined by

$$
J(u)=\left\{j \in X^{*}:\langle u, j\rangle=\|u\|^{2},\|j\|=\|u\|\right\}
$$

(see Browder [1] and Kirk and Morales [9]). Here $\langle\cdot, \cdot\rangle$ denotes the generalized duality pairing. In addition, $A$ is said to be strongly accretive if $I-A$ is strongly pseudo-contractive. For $k=1, T$ is called pseudo-contractive, while $A$ is called

Received by the editors December 3, 2003 and, in revised form, August 10, 2004.

2000 Mathematics Subject Classification. Primary 47H10.

Key words and phrases. Strongly pseudo-contractive, pseudo-contractive, compact operators. 
accretive. If $\gamma$ denotes the measure of noncompactness (for instance, see [13]), we say that a continuous mapping $g$ from $D$ into $X$ is condensing if $\gamma(g(A))<\gamma(A)$ for every bounded $A \subset D$ whose $\gamma(A)>0$. Also a continuous mapping $g$ is said to be compact if it maps bounded sets onto relatively compact sets.

In the following, we use the symbol $\partial_{K} D$ to denote the relative boundary of $D$ in $K$. We also use $B(u ; r)$ to denote an open ball centered at $u \in X$ with radius $r>0$, while $\operatorname{seg}[u, v]$ denotes the set $\{(1-t) u+t v: t \in[0,1]\}$.

\section{Preliminaries}

Proposition 1. Let $X$ be a Banach space and let $K$ be a closed convex subset of $X$. Let $D$ be an open subset of $K$ with $0 \in D$. Suppose $g: \bar{D} \rightarrow K$ is a condensing mapping satisfying the Leray-Schauder condition:

$$
g(x) \neq \lambda x \text { for } x \in \partial_{K} D \text { and } \lambda>1 .
$$

If, in addition, the set $\{x \in D: g(x)=\lambda x$ for some $\lambda>1\}$ is bounded, then $g$ has a fixed point in $\bar{D}$.

To prove this proposition we need the following Lemma, which is patterned after Lemma 1 of Petryshyn and Fitzpatrick [15].

Lemma. Let $X$ be a Banach space and let $K$ be a closed convex subset of $X$. Let $D$ be a subset of $K$, which contains a nonempty precompact subset $E$. Suppose $g: D \rightarrow K$ is a condensing mapping. Then there exists a compact and convex subset $K_{0}$ of $K$ such that $E \subset K_{0}$ and $g\left(K_{0} \cap D\right) \subset K_{0}$.

Proof of Proposition 1. Suppose there are no fixed points on $\partial_{K} D$. Define the subset $E$ of $K$ by

$$
E=\{x \in \bar{D}: x=\operatorname{tg}(x) \text { for some } t \in[0,1]\} .
$$

Since $0 \in E$ and $g$ is continuous, then $E$ is a nonempty closed subset of $K$. In addition, since $E \subset \overline{\mathrm{co}}(g(E) \cup\{0\}), E$ is also compact. Then by the previous Lemma there exists a compact and convex subset $C$ of $K$ containing $E$ such that $g(C \cap \bar{D}) \subset C$. On the other hand, since $E \cap \partial_{K} D=\emptyset$, there exists a continuous function $\mu: K \rightarrow[0,1]$ such that $\mu(x)=1$ if $x \in E$, while $\mu(x)=0$ for $x \in \partial_{K} D$. We now define $\tilde{g}: K \rightarrow K$ by $\tilde{g}(x)=\mu(x) g(x)$ if $x \in \bar{D}$; otherwise, $\tilde{g}(x)=0$. Then $\tilde{g}$ is continuous on $K$. Moreover, the restriction $\tilde{g}$ to $C$ is a compact mapping from $C$ into $C$. Therefore, it has a fixed point $x_{0} \in C$. It remains to show that $x_{0}$ is, indeed, a fixed point of $g$. To see this, we first observe that $x_{0} \in \bar{D}$; otherwise $x_{0}=0$, which is a contradiction. This means $x_{0}=\mu\left(x_{0}\right) g\left(x_{0}\right)$, and hence $x_{0} \in E$. Therefore $\mu\left(x_{0}\right)=1$, and thus $g\left(x_{0}\right)=x_{0}$.

Corollary 1. Let $K$ be a closed cone of a Banach space $X$, let $G$ be a bounded open subset of $X$ containing the origin, and let $D=G \cap K$. Suppose $g: \bar{D} \rightarrow K$ is a condensing mapping satisfying the Leray-Schauder condition:

$$
g(x) \neq \lambda x \text { for } x \in \partial_{K} D \text { and } \lambda>1 .
$$

Then $g$ has a fixed point in $\bar{D}$.

Corollary 1 extends the theorem of Gatica and Kirk [2] and Corollary 2 of Kirk [7] to cones whose interior may be empty. Additionally, Proposition 1 extends Corollary 1 of [7]. 
Corollary 2. Let $X$ be a Banach space and let $K$ be a closed convex subset of $X$ with $0 \in K$. Suppose $g: K \rightarrow K$ is a condensing mapping such that the set

$$
F:=\{x \in K: g(x)=\lambda x \text { for some } \lambda>1\}
$$

is bounded. Then $g$ has a fixed point in $K$.

Proof. Since $F$ is bounded, we select $r>0$ so that $\bar{F} \subset B(0 ; r)$. Consequently, $g$ satisfies the Leray-Schauder condition on $\partial_{K} D$ where $D=B(0 ; r) \cap K$. Therefore, by Proposition 1, $g$ has a fixed point.

\section{MAIN RESUltS}

We begin with the definition of the Hausdorff metric $H$. Let $\varepsilon>0$ and let $A$ be a bounded subset of $X$. Then

$$
N_{\varepsilon}(A)=\{x \in X: \operatorname{dist}(x, A)<\varepsilon\}
$$

denotes an $\varepsilon$-neighborhood of $A$. Suppose $A$ and $B$ are bounded subsets of $X$. We define the Hausdorff metric $H$ by

$$
H(A, B)=\inf \left\{\varepsilon: A \subset N_{\varepsilon}(B) \text { and } B \subset N_{\varepsilon}(A)\right\} .
$$

If we denote by $\mathcal{B}(X)$ the metric space of all nonempty bounded and closed subsets of $X$, then we say that a mapping $T$ from $D$ into $\mathcal{B}(X)$ is continuous at $x_{0}$ if for every $\varepsilon>0$ there exists $\delta>0$ such that

$$
H\left(T(x), T\left(x_{0}\right)\right)<\varepsilon \text { whenever }\left\|x-x_{0}\right\|<\delta .
$$

Proposition 2. Let $X$ be a Banach space and let $D$ be an open subset of $X$. Suppose $A_{t}: \bar{D} \rightarrow \mathcal{B}(X)$ is a continuous (relative to the Hausdorff metric $H$ ) and strongly accretive mapping for each $t \in[0,1]$. Suppose in addition that

$$
H\left(A_{t}(x), A_{s}(x)\right) \leq M|t-s| \text { for all } x \in \bar{D} \text {. }
$$

Then $W=\left\{(t, u): t \in[0,1]\right.$ and $\left.u \in A_{t}(D)\right\}$ is open in $[0,1] \times X$.

Proof. Let $\left(t_{0}, u_{0}\right) \in W$. Then there exists $x_{0} \in D$ such that $u_{0} \in A_{t_{0}}\left(x_{0}\right)$. For each $t \in[0,1]$, Theorem 3.1 of [8] implies that $A_{t}(D)$ is open in $X$, and thus, in particular, there exists $r>0$ such that $B\left(u_{0} ; r\right) \subset A_{t_{0}}(D)$. In addition, since $A_{t}(\bar{D})$ is closed and $A_{t}$ is a continuous one-to-one mapping, it is easily seen that $\partial A_{t}(D)=A_{t}(\partial D)$. Also, as a consequence of (1), we may choose a neighborhood $V\left(t_{0}\right) \subset[0,1]$ such that

$$
H\left(A_{t}(x), A_{t_{0}}(x)\right)<r / 3 \text { for } t \in V\left(t_{0}\right) \text { and } x \in \bar{D} .
$$

Therefore, $A_{t}(\partial D) \cap B\left(u_{0} ; r / 2\right)=\emptyset$ for $t \in V\left(t_{0}\right)$. To see this, suppose the contrary. Then there exists $x \in \partial D$ and $u \in X$ such that $u \in A_{t}(x)$ and $\left\|u-u_{0}\right\|<r / 2$. We may also select $v \in A_{t_{0}}(x)$ so that

$$
\|u-v\| \leq H\left(A_{t}(x), A_{t_{0}}(x)\right)+r / 6
$$

(see Nadler [12]), which implies that $\left\|v-u_{0}\right\| \leq\|v-u\|+\left\|u-u_{0}\right\|<r$. Therefore, $v \in A_{t_{0}}(D)$, a contradiction! On the other hand, we may choose $u_{1} \in A_{t}\left(x_{0}\right)$ such that

$$
\left\|u_{1}-u_{0}\right\| \leq H\left(A_{t}\left(x_{0}\right), A_{t_{0}}\left(x_{0}\right)\right)+r / 6<r / 2,
$$

implying that $B\left(u_{0} ; r / 2\right) \cap A_{t}(D) \neq \emptyset$. Therefore, $B\left(u_{0} ; r / 2\right) \subset A_{t}(D)$; otherwise, if it did exist $w \in B\left(u_{0} ; r / 2\right) \backslash A_{t}(D)$, and then

$$
\operatorname{seg}\left[u_{1}, w\right] \cap \partial A_{t}(D) \neq \emptyset
$$


which contradicts what we stated above. This means that $B\left(u_{0} ; r / 2\right) \subset A_{t}(D)$ for all $t \in V\left(t_{0}\right)$, and hence the open set $V\left(t_{0}\right) \times B\left(u_{0} ; r / 2\right) \subset W$.

Theorem 1. Let $X$ be a Banach space and let $D$ be an open subset of $X$. Suppose $T: \bar{D} \rightarrow \mathcal{B}(X)$ is a continuous and strongly pseudo-contractive mapping (with constant $k$ ) and $g: \bar{D} \rightarrow X$ is a compact mapping with $g(D)$ bounded. Suppose there exists $z \in D$ such that

$$
\lambda(x-z) \notin T(x)-z+g(x) \text { for } x \in \partial D \text { and } \lambda>1 .
$$

Then $T+g$ has a fixed point in $\bar{D}$.

Proof. By replacing $T$ with $T(x+z)-z, g(x)$ with $g(x+z)$ and $D$ with $D-z$, we may assume that $z=0$ in (2). We first observe that the set

$$
E=\{x \in D: \lambda x \in T(x)+g(x) \text { for some } \lambda>1\}
$$

is bounded. To see this, let $\lambda x=u+g(x)$ for some $u \in T(x)$ and $\lambda>1$. Then there exists $j \in J(x)$ such that

$$
\begin{aligned}
\langle u-v, j\rangle & =\langle\lambda x-g(x)-v, j\rangle \\
& \leq k\|x\|^{2}
\end{aligned}
$$

where $v \in T(0)$. Then this implies

$$
(\lambda-k)\|x\| \leq\|g(x)+v\|,
$$

and since $g(D)$ is bounded, hence so is $E$. Let $t \in[0,1]$. Then $I-t T$ is continuous and strongly accretive, and hence $(I-t T)(D)$ is open in $X$. Now define

$$
U=\{(t, u): t \in[0,1] \text { and } u \in(I-t T)(D)\} .
$$

Then by Proposition 2, $U$ is open in $K:=[0,1] \times X$. We should observe that $K$ is a closed and convex subset of the Banach space $\mathbb{R} \times X$ with norm $\|(t, u)\|_{1}=|t|+\|u\|$. Since $I-t T$ is also expansive, then the mapping $h_{t}:(I-t T)(\bar{D}) \rightarrow \bar{D}$ defined by $h_{t}(x)=(I-t T)^{-1}(x)$ is single-valued and satisfies

$$
\left\|h_{t}(x)-h_{t}(y)\right\| \leq \frac{1}{1-t k}\|x-y\| \leq \frac{1}{1-k}\|x-y\| .
$$

We now define the mappings $h: \bar{U} \rightarrow[0,1] \times \bar{D}$ and $f:[0,1] \times \bar{D} \rightarrow K$ by $h(t, x)=$ $\left(1, h_{t}(x)\right)$ and $f(t, x)=(t, g(x))$ respectively. Then the composition mapping $f \circ h$ maps $\bar{U}$ into $K$. In addition,

$$
\begin{aligned}
\|h(t, x)-h(s, y)\|_{1} & \leq\|h(t, x)-h(t, y)\|_{1}+\|h(t, y)-h(s, y)\|_{1} \\
& \leq\left\|h_{t}(x)-h_{t}(y)\right\|+\left\|h_{t}(y)-h_{s}(y)\right\| \\
& \leq \frac{1}{1-t k}[\|x-y\|+\|u\||t-s|]
\end{aligned}
$$

for some $u \in T\left(h_{s}(y)\right)$. Then $h$ is a continuous and bounded operator on $\bar{U}$. On the other hand, since

$$
\|f(t, x)-f(s, y)\|_{1} \leq|t-s|+\|g(x)-g(y)\|
$$

and $g$ is compact, then so is $f$. Therefore $f \circ h$ is also compact. We shall show now that, indeed, $f \circ h$ satisfies the $(L-S)$ condition on $\partial_{K} U$. To see this, let $(t, x) \in \partial_{K} U$ and $\lambda>1$ such that $f(h(t, x))=\lambda(t, x)$. Then

$$
f\left(1, h_{t}(x)\right)=\left(1, g\left(h_{t}(x)\right)\right)=(\lambda t, \lambda x) .
$$


This implies that $g\left(h_{t}(x)\right)=\lambda x$, while $\lambda t=1$. Since $\lambda>1$, then $x \in \partial(I-t T)(D)$. This means there exists $w \in \partial D$ such that $x \in w-t T(w)$. Hence $w=h_{t}(x)$ and thus $g(w)=\lambda x$, which implies that $\lambda w \in T(w)+g(w)$, contradicting (11). Also, since $E$ is bounded, the above argument allows us to derive that the set

$$
M=\{(t, x) \in U: f(h(t, x))=\lambda(t, x) \text { for some } \lambda>1\}
$$

is bounded. Therefore, by Proposition 1, $f \circ h$ has a fixed point $(t, x) \in \bar{U}$, and hence $t=1$ and $g\left(h_{1}(x)\right)=x$. Now by letting $u=h_{1}(x)$, we obtain $u \in T(u)+g(u)$, which completes the proof.

Theorem 1] is an extension of Theorem 2.1 of [14] to multivalued mappings with a nondegree theory argument under no boundedness assumption on $T(D)$. We should also mention the proof of Theorem 2.1, implicitly, uses that $g(D)$ is relatively compact. This fact does not hold unless $D$ itself is bounded. We include an example to illustrate this behavior.

Example. Let $X$ be the real Hilbert space $l_{2}$ and let $g: l_{2} \rightarrow l_{2}$ be defined by

$$
g(x)= \begin{cases}\left(x_{1}, 0, \ldots\right) & \text { if }\|x\| \leq 1, \\ \frac{1}{\|x\|}\left(x_{1}, \ldots, x_{n},(\|x\|-n) x_{n+1}, 0, \ldots\right) & \text { if } n \leq\|x\| \leq n+1, n \in \mathbb{N} .\end{cases}
$$

Then $g$ is clearly compact. However, the sequence $\left\{g\left(n e_{n}\right)\right\}_{n=1}^{\infty}$, where $\left\{e_{n}\right\}$ is the canonical basis for $l_{2}$, is not relatively compact. Therefore $g\left(l_{2}\right)$ is bounded but not relatively compact.

Theorem 2. Let $X$ be a Banach space and let $D$ be a bounded open subset of $X$. Suppose $A: \bar{D} \rightarrow \mathcal{B}(X)$ is a continuous accretive operator and $g: \bar{D} \rightarrow X$ is compact. Suppose there exists $z \in D$ such that

$$
t(x-z) \notin A(x)+g(x) \text { for } x \in \partial D \text { and } t<0 .
$$

Then $0 \in \overline{\mathcal{R}(A+g)}$.

Proof. As before, by replacing $A(x)$ by $A(x+z), g(x)$ with $g(x+z)$ and $D$ by $D-z$, one may take $z=0$ in (3). Now define

$$
T(x)=(1-\eta) x-A(x) \text { for } x \in \bar{D} \text { and } \eta>0 .
$$

Then $T-g$ satisfies the Leray-Schauder condition (see (2) above with $z=0$ ). Since $T$ is strongly pseudo-contractive and $-g$ is compact, then, by Theorem 1 $T-g$ has a fixed point in $\bar{D}$. This means there exists $x \in \bar{D}$ such that

$$
0 \in \eta x+A(x)+g(x) \text { for each } \eta>0 \text {. }
$$

Since $D$ is bounded, we may choose $\eta \rightarrow 0$, and conclude $0 \in \overline{\mathcal{R}(A+g)}$.

As a consequence of Theorem 2 we derive Theorem 7 of Kartsatos [5].

Corollary 3. Let $X$ be a Banach space and let $D$ be a bounded open subset of $X$ with $0 \in D$. Suppose $A: \bar{D} \rightarrow \mathcal{B}(X)$ is continuous and accretive, while $g \in \bar{D} \rightarrow X$ is compact. Suppose for each $x \in \partial D$ there exists $j \in J(x)$ such that

$$
\langle u+g(x)-w, j\rangle \geq 0 \text { for } u \in T(x) .
$$

Then $w \in \overline{\mathcal{R}(A+g)}$. 
Proof. We first observe the operator $A+g-w$, for a fixed $w \in X$, satisfies condition (3). To see this, let $x \in \partial D$ and suppose $t x \in A(x)+g(x)-w$ for some $t \in \mathbb{R}$. Then, by (4), $t\|x\|^{2} \geq 0$. Hence $t>0$. Therefore (3) holds. Since $A-w$ is also accretive, then Theorem 2 completes the proof.

We remark that Corollary 3 extends Theorem 7 (ii) of $[5$ to multivalued accretive operators, and also under a weaker boundary condition. Indeed, (4) holds for a single functional $j \in J(x)$, contrary to [5, where it is assumed that (4) must hold for all $j \in J(x)$. Additionally, see [6], for a complete recollection on compact perturbations.

\section{ACKNOWLEDGMENT}

The author wishes to thank his colleague Leigh Lunsford for suggesting the above mentioned example.

\section{REFERENCES}

[1] F. E. Browder, Nonlinear operators and nonlinear equations of evolution in Banach spaces, Proc. Symp. Pure Math. 18 AMS (1976). MR0405188 (53:8982)

[2] J. A. Gatica and W. A. Kirk, A fixed point theorem for $k$-set-contractions defined in a cone, Pacific J. Math. 53 (1974), 131-136. MR0353071 (50:5557)

[3] N. Hirano, Some surjectivity theorems for compact perturbations of accretive operators, Nonlinear Analysis 8 (1984), 765-774. MR0750049 (86a:47054)

[4] A. G. Kartsatos, Mapping theorems involving compact perturbations and compact resolvents of nonlinear operators in Banach spaces, J. Math. Anal. Appl. 80 (1981), 130-146. MR0614247 (82e:47085)

[5] A. G. Kartsatos, On compact perturbations and compact resolvents of nonlinear m-accretive operators in Banach spaces, Proc. Amer. Math. Soc. 119 (1993), 1189-1199. MR1216817 (94c:47076)

[6] A. G. Kartsatos, Recent results involving compact perturbations and compact resolvents of accretive operators in Banach spaces, World Congress in Nonlinear Analysts 1992, Vol. I-IV (Tampa, FL, 1992), 2197-2222, de Gruyter, Berlin, 1996. MR1389246

[7] W. A. Kirk, A remark on condensing mappings, J. Math. Anal. Appl. 51 (1975), 629-632. MR0380526 (52:1426)

[8] W. A. Kirk, Local expansions and accretive mappings, Internat. J. Math. \& Math. Sci. 6 (1983), 419-429. MR0712561 (85b:47061)

[9] W. A. Kirk and C. H. Morales, Fixed point theorems for local strong pseudo-contractions, Nonlinear Anal. Theory Methods Appl. 4 (1980), 363-368. MR0563815 (81a:47056)

[10] C. H. Morales, Pseudo-contractive mappings and the Leray-Schauder boundary condition, Comment. Univ. Carolinae 20 (1979), 745-756. MR0555187 (80k:47067)

[11] C. H. Morales, Existence theorems for strongly accretive operators in Banach spaces, SIMAA 4 (2002) Taylor \& Francis, 361-368. MR 1938855 (2003k:47081)

[12] S. B. Nadler, Jr., Multi-valued contraction mappings, Pacific J. Math. 30 (1969), 475-488. MR0254828 (40:8035)

[13] Roger D. Nussbaum, The fixed point index for local condensing maps, Ann. Mat. Pura Appl. 89 (1971), 217-258. MR0312341 (47:903)

[14] D. O'Regan, Fixed point theory for compact perturbations of pseudocontractive maps, Archivum Mathematicum 34 (1998), 401-415. MR1662064 (99j:47092)

[15] W. V. Petryshyn and P. M. Fitzpatrick, Fixed point theorems for multivalued noncompact inward maps, J. Math. Anal. Appl. 46 (1974), 456-767. MR0361787 (50:14232)

Department of Mathematics, University of Alabama in Huntsville, Huntsville, Alabama 35899

E-mail address: morales@math.uah.edu 